American J. of Engineering and Applied Sciences 4 (1): 133-141, 2011

ISSN 1941-7020

(C) 2010 Science Publications

\title{
Fractional Quantum Field Theory on Multifractals Sets
}

\author{
El-Nabulsi Ahmad Rami \\ Department of Nuclear and Energy Engineering, Cheju National University, \\ Ara-dong 1, Jeju 690-756, South Korea
}

\begin{abstract}
Problem statement: This study is a contribution to the general program of describing complex dynamical systems using the tool of fractional calculus of variations. Approach: Following our previous work, fractional quantum field theory based on the fractional actionlike variational approach supported by Saxena-Kumbhat fractional integrals functionals, fractional derivative of order $(\alpha, \beta)$ and dynamical fractional exponent on multi-fractal sets is considered. Results: In order to build the required theory, we introduce the Saxena-Kumbhat hypergeometric fractional functionals determined on the functions on a multifractal sets. We prove, developing the corresponding fractional calculus of variations, that a hierarchy of differential equations can be developed from the extended fractional Lagrangian formalism. Besides, a generalization of the resulting Hamiltonian and Lagrangian dynamics on the complex plane is addressed. Conclusion: The new complexified dynamics guides to a new dynamics which may differ totally from the classical mechanics cardinally and may bring new appealing consequences. Some additional interesting results are explored and discussed in some details.
\end{abstract}

Key words: Fractional action-like variational approach, multifractal sets, Euler-Lagrange equations, Saxena-Kumbhat fractional integral, fractional derivative, dynamical fractional exponent

\section{INTRODUCTION}

Fractional dynamics is the study of complex dynamical systems that can be cast in terms of solutions to fractional differential equations to which the fractional calculus can be correctly applied. Fractional calculus has become an exciting new mathematical method of solution of diverse problems in mathematics, science and engineering. Making use of fractional derivatives and integrals, one may describe more accurately a complex system and accordingly investigate more completely its dynamical and physical properties. Although fractional calculus has been studied for over 300 years, it has been regarded principally as a mathematical curiosity until about 1992, where fractional dynamical equations were pretty much restricted to the realm of mathematics and engineering including hydrology, viscoelastivity, heat conduction, polymer physics, chaos and EL-NABULSI Ahmad Rami fractals, control theory, plasma physics, wave propagation in complex and porous media, astrophysics, cosmology, quantum field theory, potential theory and so on (Aghaei et al., 2009; Samko et al., 1993; Miller and Ross, 1993; Podlubny, 1999; Hilfer, 2000; Kluwer, 2004; Ortigueira and Machado, 2006; 2008; El-Nabulsi, 2008a; 2009a).

Actually, there exist numerous different forms of fractional integral and derivatives operators and the definition of the fractional order derivative and integral are not unique where several definitions exist, e.g., Grunwald-Letnikov, Caputo, Weyl, Feller, ErdelyiKober, Riesz, Saxena, Kumbhat, Kiryakova, Srivastava and Raina.

A theme of present strong research concerns the study fractional Lagrangian and Hamiltonian dynamics systems based on the fractional problems of the Calculus Of Variations (COV) (Ali et al., 2009). Different forms of Euler-Lagrange equations were obtained in the literature depending on the action and type of fractional derivative used. The formulation of the fractional problems of the COV still needs more elaboration as the problem is deeply related to the fractional quantization procedure and to the presence of non-local fractional differential operators. Many problems also occur, e.g.:

- The occurrence of non-local fractional differential operators,

- The adjoint of a fractional differential operator used to describe the dynamics is not the negative of itself

- The derived Euler-Lagrange equations depend on left and right fractional derivatives even when the dynamics depend only on one of them

- The appearance of a very complicated Leibniz rule (the derivative of product of functions) 
- The non-presence of any fractional analogue of the chain rule.

One possible simple and realistic approach to better model non-conservative and weak dissipative complex dynamical systems is the Fractional Action-Like Variational Approach (FALVA) based on the concept of left Riemann-Liouville fractional integral functionals with one parameter $\alpha$ but not on fractional-order derivatives of the same order (El-Nabulsi, 2005a; 2005b). Many encouraging results were successfully obtained and discussed (El-Nabulsi and Torres, 2008; 2007; El-Nabulsi, 2007; 2008b; 2008c; 2009b; 2009c; 2009d; 2009e; 2009f; 2009g). In order to stimulate more interest in the subject and to show its utility, this contribution is devoted to a new generalization of the FALVA by introducing mainly inside the fractional action-like integral the Saxena-Kumbhat hypergeometric fractional operator, augmented by a fractional derivative and a dynamical fractional exponent defined on multifractal time and space sets, in particular when the fractional dimensions of time and space are dynamical, e.g., $d_{i}=1+\varepsilon\left(\vec{x}_{i}\right)$. This notion is based on the Mandelbrot ideas of the fractal geometry of nature and is expected to work on a small multifractal intervals set $\mathrm{S}_{\mathrm{i}}$ which is build from multifractal subsets $\mathrm{S}\left(\overrightarrow{\mathrm{x}}_{\mathrm{i}}\right)$ (Al-Daoud, 2008; Kobolev, 2000). Each of them consists in fact of a continuous, but not differentiable bounded set of small elements. Both time and space are considered as the only material fields existing in the manifold and thus generating all other physical fields. In this work, we suppose that the fractal dimensions are slightly different from unity and hence valid for small densities of Lagrangians in points $\overrightarrow{\mathrm{x}}_{\mathrm{i}}$. The function $\mathrm{d}_{\mathrm{i}}=\mathrm{d}\left(\overrightarrow{\mathrm{x}}_{\mathrm{i}}\right)$ is expected to be continuous and describes the fractional dimensions.

EL-NABULSI Ahmad Rami: Here, we cite our motivations for introducing the hypergeometric functions, the fractional derivatives and a dynamical fractional exponent respectively:

- The motivation for introducing the hypergeometric function in the fractional action is that the later is intimately associated with the Laplace and wave equations in four-dimensional space and their extensions. Therefore, we expect that the new formalism will lead to a generalization of the dynamical differential equations as its presence in the differential equations will leads to an extremely rich structure as the hypergeometric function contains almost all special functions (orthogonal, power-type and elliptic integrals) as a particular or limiting case

- Our motivation for introducing the fractional derivative came from the fact that the later portray more precisely the non-trivial behavior of complex physical systems whose dynamics are distant from equilibrium, i.e. dealing with fractional derivatives is not more complex than with usual differential operators. Dynamical equations in fractional derivatives describe normally the evolution of physical systems with loss, the fractional exponent of the derivative being a measure of the fraction of the states of the dynamical system that are preserved during the evolution time. Amazingly, fractional differential operators are in reality global (non-local) operators and limits in the sense of ultra-long time. For that main reason, dynamical systems with fractional order are non-conservative and hence the fractional derivative is broadly used for describing intermediary physical processes and critical phenomena in non-equilibrium complex non-linear systems

- Time-dependent fractional exponent plays a significant role in diverse branches of complex dynamical systems, e.g. self-affine time-sequential data (Sabanal and Nakagawa, 1995)

Generalized FALVA augmented by the saxenakumbhat hypergeometric fractional operator, fractional derivative and time-dependent fractional dimensions: In order to construct the required theory, it is desirable to characterize the fractional functionals determined on the functions, given on a multifractal sets. We start by recalling the following definition:

Definition 1: The Saxena-Kumbhat hypergeometric fractional integrals for a function $f \in L_{p}(0, \infty)$ are defined by (Samko et al., 1993):

$$
\begin{aligned}
& I_{\text {Left }} f(t) \triangleq \frac{1}{\Gamma(\alpha)} t^{-\alpha-\sigma} \int_{0}^{t} f(\tau) \tau^{\sigma}(t-\tau)^{\alpha-1}{ }_{2} F_{1}\left(a, b ; c ; \zeta\left(1-\frac{\tau}{t}\right)\right) d \tau \\
& I_{\text {Right }} f(t) \triangleq \frac{1}{\Gamma(\alpha)} t^{\delta} \int_{t}^{\infty} f(\tau) \tau^{-\delta-\alpha}(\tau-t)^{\alpha-1}{ }_{2} F_{1}\left(a, b ; c ; \zeta\left(1-\frac{\tau}{t}\right)\right) d \tau
\end{aligned}
$$

EL-NABULSI Ahmad Rami: Here ${ }_{2} \mathrm{~F}_{1}$ is the Gaussian hypergeometric function defined through its power series expansion around $\mathrm{y}=0$ by:

$$
\begin{aligned}
& { }_{2} F_{1}(a, b ; c ; y)=\sum_{n=0}^{+\infty} \frac{(a)_{n}(b)_{n}}{(c)_{n} n !} y^{n}=\sum_{n=0}^{+\infty} t_{n} y^{n} \\
& t_{1}=1 \text { and: }
\end{aligned}
$$


$t_{n+1}=\frac{(a+n)(b+n)}{(c+n)(n+1)} t_{n}, n \geq 0$

and where $(x)_{n}=1 \cdot x \cdot \ldots \cdot(x+n-1)$ is standard Appell or Pochhammer symbol, $\quad \mathfrak{R}(\alpha)>0, \quad \mathfrak{R}(\sigma)>-1 / \mathrm{q}$, $\Re(\delta)>-1 / p, \quad \mathrm{p}^{-1}+\mathrm{q}^{-1}=1, \quad \mathrm{p} \geq 1, \quad \mathrm{c} \neq 0,-1,-2, \ldots$, $\mathfrak{R}(\mathrm{c}-\mathrm{b}-\mathrm{a})>0$ and $|\arg (1-\zeta)|<\pi$. Normally, the series always converges for $|y|<1$ (Granik, 2004).

Remark 1: As it is well-known, many problems in physics and mathematical physics require a conformal symmetry groups which in its turn accounts for many of the properties of ${ }_{2} \mathrm{~F}_{1}$. It is a fundamental property of any dynamical phenomena or physical process. It is noteworthy that ${ }_{2} \mathrm{~F}_{1}$ plays a crucial role in many branches of science, including atomic physics, (integrals in atomic collision are very often analytically expressed with the ${ }_{2} \mathrm{~F}_{1}$ function), celestial dynamics (the 3-body problem), astrophysics (in particular in linearized MHD equations), General Relativity (gravitational waves emitted by binary stars). Furthermore, one of the most powerful techniques for calculating Feynman diagrams is based on their presentation in terms of hypergeometric functions. Such a representation can be used for numerical evaluation, construction of the asymptotic expansion (Bitev et al., 2009). It can also provide analytical wave function solutions of the Schrödinger equation and of the relativistic KleinGordon and Dirac equations (Michel and Soitsov, 2008; Gomes and Oliveira, 2004).

Remark 2: For mathematical simplicity, we will define the new variable $X \triangleq 1-(\tau / t) \in \mathbb{R}$, i.e., we will perform our calculation in the real plane and we set $\zeta=1$ for mathematical simplicity. As the fractional problem of the $\mathrm{CoV}$ is based on the left fractional integral, the Saxena-Kumbhat hypergeometric fractional left integral for a function $f \in L_{p}(0, \infty)$ with respect to the new variable is now defined on the interval by:

$\mathrm{I}_{\text {Left }} \mathrm{f} \triangleq \frac{1}{\Gamma(\alpha)} \int_{0}^{1} \mathrm{f}(\mathrm{t}(1-\mathrm{X}))(1-\mathrm{X})^{\alpha+\sigma-1}{ }_{2} \mathrm{~F}_{1}(\mathrm{a}, \mathrm{b} ; \mathrm{c} ; \mathrm{X}) \mathrm{dX}$

We may propose the following problem.

Problem 1: Consider a smooth n-dimensional manifold $\mathrm{M}$ and let $\mathrm{L}: \mathrm{C}^{1}\left([0,1] \times \mathbb{R}^{\mathrm{n}} \times \mathbb{R} ; \mathbb{R}\right)$ be the smooth Lagrangian function satisfying fixed boundary conditions $\mathrm{q}(0)=\mathrm{q}_{0}$ and $\mathrm{q}(1)=\mathrm{q}_{1}$. Find the stationary points of the integral functional on multifractal time and space sets:

$$
\begin{aligned}
& \left.\mathrm{S}[\mathrm{q}(\bullet)]=\int_{0}^{1} \mathrm{~L}(1-\mathrm{X})\right) \frac{(1-X)^{\alpha(X)+\sigma(X)-1}}{\Gamma(\alpha(\mathrm{t}(1-\mathrm{X})))} \\
& { }_{2} \mathrm{~F}_{1}(\mathrm{a}, \mathrm{b} ; \mathrm{c} ; \mathrm{X}) \mathrm{e}^{\chi \cdot \mathrm{t}(1-\mathrm{X})} \mathrm{q}(\mathrm{t}(1-\mathrm{X})), \mathrm{q}(\mathrm{t}(1-\mathrm{X})),
\end{aligned}
$$

EL-NABULSI Ahmad Rami: $X \neq 1, \chi \in \mathbb{R}, q^{\prime}=$ $\mathrm{dq} / \mathrm{dX}$ and the smooth Lagrangian function $\mathrm{L}(\dot{\mathrm{q}}(\mathrm{t}(1-\mathrm{X})), \mathrm{q}(\mathrm{t}(1-\mathrm{X})), \mathrm{t}(1-\mathrm{X})) \quad$ is actually weighted with $(1-\mathrm{X})^{\alpha(\mathrm{X})+\sigma(\mathrm{X})-1}{ }_{2} \mathrm{~F}_{1}(\mathrm{a}, \mathrm{b} ; \mathrm{c} ; \mathrm{X}) / \Gamma(\alpha(\mathrm{t}(1-\mathrm{X})))$. Here, the fractional derivative operator of order $(\alpha$, $\beta), a, b, t \in \mathbb{R}, a<t$ is defined by Cresson (2007):

$$
\begin{aligned}
D_{(\gamma)}^{(\alpha, \beta)} f(\bullet) & =\frac{1}{2}\left[D_{\left(a_{+}\right)}^{(\alpha)} f(\bullet)-D_{\left(b_{-}\right)}^{(\beta)} f(\bullet)\right] \\
& +\frac{i \gamma}{2}\left[D_{\left(a_{+}\right)}^{(\alpha)} f(\bullet)+D_{\left(b_{-}\right)}^{(\beta)} f(\cdot)\right]
\end{aligned}
$$

$\gamma \in \mathbb{C}, i=\sqrt{-1}$ and $0<\alpha, \beta<1$

Here:

$$
\begin{aligned}
& D_{\left(a_{+}\right)}^{(\alpha)} f(t(1-X))=\frac{t^{1-\beta}}{\Gamma(1-\beta)} \frac{\partial}{\partial t} \int_{0}^{1-a / t} f(t(1-X)) X^{-\beta} d X \\
& D_{\left(a_{-}\right)}^{(\beta)} f(t(1-X))=-\frac{t^{1-\beta}}{\Gamma(1-\beta)} \frac{\partial}{\partial t} \int_{1-b / t}^{0} f(t(1-X)) X^{-\beta} d X
\end{aligned}
$$

and:

$$
\Gamma(\alpha(t(1-X)))=t^{\alpha(t(1-X))} \int_{-\infty}^{1}(1-X)^{\alpha(t(1-X))-1} \exp (-t(1-X)) d X
$$

is the modified Euler gamma function.

Remark 3: The following properties hold:

$$
\begin{aligned}
& \int_{a}^{b} D_{(\gamma)}^{(\alpha, \beta)} f(X) g(X) d X=-\int_{a}^{b} f(X) D_{-(\gamma)}^{(\beta, \alpha)} g(X) d t, a \leq X \leq b \\
& \int_{a}^{b} D_{\left(a_{+}\right)}^{(\alpha)} f(X) g(X) d X=(-1)^{\alpha} \int_{a}^{b} f(X) D_{\left(b_{-}\right)}^{(\alpha)} g(X) d X
\end{aligned}
$$

$$
\begin{aligned}
& D_{(a)}^{(\alpha)} D_{(a)}^{(\beta)} f(X)=D_{(a)}^{(\alpha+\beta)} f(X)-\left.\sum_{i=1}^{k} D_{(a)}^{(\sigma-i)} f(X)\right|_{X=a} \frac{(t-a)^{-\alpha-i}}{\Gamma(1-\alpha-i)}, \\
& 0 \leq k-1 \leq q \leq k
\end{aligned}
$$


provided that $\mathrm{f}(\mathrm{a})=\mathrm{f}(\mathrm{b})=0$ and $\mathrm{g}(\mathrm{a})=\mathrm{g}(\mathrm{b})=0$. $\mathrm{k}$ in Eq. 11 is a whole number.

Remark 4: Since in our approach we have defined fractional differentiation through integration, fractional derivatives are no longer local operations. They are only defined over an interval. This may explain why during the early time, Leibnitz believed it was an absurdity since his objective was to obtain a unique and local derivative.

Theorem 1: If $\mathrm{q}(\mathrm{t}(1-\mathrm{X}))$ are solutions to the previous problem, then it satisfies the following generalized fractional Euler-Lagrange equation:

$$
\begin{aligned}
& \frac{\partial \mathrm{L}\left(\mathrm{D}_{(\gamma)}^{(\alpha, \beta)} \mathrm{q}(\mathrm{t}(1-\mathrm{X})), \mathrm{q}(\mathrm{t}(1-\mathrm{X})), \mathrm{t}(1-\mathrm{X})\right)}{\partial \mathrm{q}}- \\
& \mathrm{D}_{-(\gamma) ; \mathrm{X}}^{(\beta, \alpha)}\left(\frac{\partial \mathrm{L}\left(\mathrm{D}_{(\gamma)}^{(\alpha, \beta)} \mathrm{q}(\mathrm{t}(1-\mathrm{X})), \mathrm{q}(\mathrm{t}(1-\mathrm{X})), \mathrm{t}(1-\mathrm{X})\right)}{\partial \mathrm{q}_{\mathrm{X}}}\right) \\
& =\left[\begin{array}{l}
{\left[\frac{\mathrm{d} \alpha}{\mathrm{dX}}+\frac{\mathrm{d} \sigma}{\mathrm{dX}}\right] \ln (1-\mathrm{X})-\frac{\alpha(\mathrm{X})+\sigma(\mathrm{X})-1}{1-\mathrm{X}}-} \\
\frac{1}{\mathrm{t}} \frac{1}{\Gamma(\alpha(\mathrm{t}(1-\mathrm{X})))} \frac{\mathrm{d}}{\mathrm{dX}}\left(\frac{1}{\Gamma(\alpha(\mathrm{t}(1-\mathrm{X})))}\right)-\chi \mathrm{t}
\end{array}\right] \\
& \times \frac{\partial \mathrm{L}\left(\mathrm{D}_{(\gamma)}^{(\beta, \alpha)} \mathrm{q}(\mathrm{t}(1-\mathrm{X})), \mathrm{q}(\mathrm{t}(1-\mathrm{X})), \mathrm{t}(1-\mathrm{X})\right)}{\partial \mathrm{q}_{\mathrm{X}}}
\end{aligned}
$$

\section{EL-NABULSI Ahmad Rami:}

$$
+\frac{1}{{ }_{2} F_{1}} \frac{\mathrm{d}_{2} \mathrm{~F}_{1}}{\mathrm{dX}} \frac{\partial \mathrm{L}\left(\mathrm{D}_{(\gamma)}^{(\beta, \alpha)} \mathrm{q}(\mathrm{t}(1-\mathrm{X})), \mathrm{q}(\mathrm{t}(1-\mathrm{X})), \mathrm{t}(1-\mathrm{X})\right)}{\partial \mathrm{q}_{\mathrm{X}}}
$$

Proof: We execute a small perturbation of the generalized coordinates:

$$
\mathrm{q}(\bullet) \rightarrow \mathrm{q}(\bullet)+\varepsilon \mathrm{h}(\bullet), \varepsilon<<1
$$

for which:

$$
\mathrm{D}_{(\gamma)}^{(\alpha, \beta)}(\mathrm{q}(\bullet)+\varepsilon \mathrm{h}(\bullet)) \approx \mathrm{D}_{(\gamma)}^{(\alpha, \beta)} \mathrm{q}(\bullet)+\varepsilon \mathrm{D}_{(\gamma)}^{(\alpha, \beta)} \mathrm{h}(\bullet)
$$

\section{Consequently:}

$$
\begin{aligned}
& \mathrm{S}[\mathrm{q}+\varepsilon \mathrm{h}]=\int_{0}^{1} \mathrm{~L}\left(\mathrm{D}_{(\gamma)}^{(\alpha, \beta)} \mathrm{q}(\mathrm{t}(1-\mathrm{X}(1-\mathrm{X}))+\varepsilon \mathrm{ch}(\mathrm{t}(1-\mathrm{X})), \mathrm{t}(1-\mathrm{X}))\right. \\
& \times \frac{(1-\mathrm{X}))^{\alpha(\mathrm{X})+\sigma(\mathrm{X})-1} \mathrm{q}(\mathrm{t}(1-\mathrm{X})),}{\Gamma(\alpha(\mathrm{t}(1-\mathrm{X})))}{ }_{2} \mathrm{~F}_{1}(\mathrm{a}, \mathrm{b} ; \mathrm{c} ; \mathrm{X}) \mathrm{e}^{\chi \cdot \mathrm{t}(1-\mathrm{X})} \mathrm{dX}
\end{aligned}
$$

which implies doing a Taylor expansion of $\mathrm{L}\left(\mathrm{D}_{(\gamma)}^{(\alpha, \beta)} \mathrm{q}(\mathrm{t}(1-\mathrm{X}))+\varepsilon \mathrm{D}_{(\gamma)}^{(\alpha, \beta)} \mathrm{h}(\mathrm{t}(1-\mathrm{X})), \mathrm{q}(\mathrm{t}(1-\mathrm{X})) \quad\right.$ in $\quad \varepsilon$ $+\varepsilon h(t(1-X)), t(1-X))$

around zero and integrating by parts:
$\mathrm{S}[\mathrm{q}+\varepsilon \mathrm{h}]=\mathrm{S}[\mathrm{q}]$

$-\varepsilon \int_{0}^{1} \int_{0}^{-\frac{\partial \mathrm{L}\left(\mathrm{D}_{(\gamma)}^{(\alpha, \beta)} \mathrm{q}(\mathrm{t}(1-\mathrm{X})), \mathrm{q}(\mathrm{t}(1-\mathrm{X})), \mathrm{t}(1-\mathrm{X})\right)}{\partial \mathrm{q}}}$

${ }_{2} \mathrm{~F}_{1}(\mathrm{a}, \mathrm{b} ; \mathrm{c} ; \mathrm{X}) \mathrm{e}^{\mathrm{x} \cdot \mathrm{t}(1-\mathrm{X})} \mathrm{h}(\mathrm{t}(1-\mathrm{X}))$

$+\left(\frac{(1-\mathrm{X})^{\alpha(\mathrm{X})+\sigma(\mathrm{X})-1}}{\Gamma(\alpha(\mathrm{t}(1-\mathrm{X})))} \times{ }_{2} \mathrm{~F}_{1}(\mathrm{a}, \mathrm{b} ; \mathrm{c} ; \mathrm{X}) \mathrm{e}^{\chi \mathrm{X} \cdot \mathrm{t}(1-\mathrm{X})}\right.$

$\left.\frac{\partial \mathrm{L}\left(\mathrm{D}_{(\gamma)}^{(\alpha, \beta)} \mathrm{q}(\mathrm{t}(1-\mathrm{X})), \mathrm{q}(\mathrm{t}(1-\mathrm{X})), \mathrm{t}(1-\mathrm{X})\right)}{\partial \mathrm{q}_{\mathrm{X}}} \mathrm{D}_{(\gamma), \mathrm{X}}^{(\alpha, \beta)} \mathrm{h}(\mathrm{t}(1-\mathrm{X}))\right)$

$+\left(\frac{(1-\mathrm{X})^{\alpha(\mathrm{X})+\sigma(\mathrm{X})-1}}{\Gamma(\alpha(\mathrm{t}(1-\mathrm{X})))} \times{ }_{2} \mathrm{~F}_{1}(\mathrm{a}, \mathrm{b} ; \mathrm{c} ; \mathrm{X}) \mathrm{e}^{\mathrm{X} \cdot \mathrm{t}(1-\mathrm{X})}\right.$

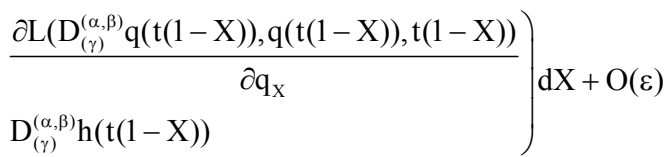

Making use of the least action principle and property (9) we arrive to (12) after simple algebra.

Remark 5: One may substitute into Eq. 12 the third term inside the bracket by the digamma function defined as the logarithmic derivative of the gamma function (Qi and Guo, 2009):

$\frac{1}{\Gamma(\alpha(t(1-X)))} \frac{d}{d X}\left(\frac{1}{\Gamma(\alpha(t(1-X)))}\right)=$

$\frac{\mathrm{d}}{\mathrm{dX}} \ln \Gamma(\alpha(\mathrm{t}(1-\mathrm{X})))=\psi(\mathrm{t}(1-\mathrm{X}))$

which it is the first of the polygamma functions and has the integral representation:

$\psi(t(1-X))=-\gamma-\frac{1}{t(1-X)}+t(1-X) \sum_{k=1}^{\infty} \frac{1}{k(t(1-X)+k)}$

$\gamma$ is the Euler-Mascheroni constant defined by:

\section{EL-NABULSI Ahmad Rami:}

$\gamma=\lim _{\mathrm{n} \rightarrow \infty}\left(\sum_{\mathrm{k}=1}^{\mathrm{n}} \frac{1}{\mathrm{k}}-\log \mathrm{n}\right) \approx 0.57721566490153286 \ldots$

Corollary 1: In terms of the polygamma function, the generalized fractional Euler-Lagrange equation is written as follows: 


$$
\begin{aligned}
& \frac{\partial \mathrm{L}\left(\mathrm{D}_{(\gamma)}^{(\alpha, \beta)} \mathrm{q}(\mathrm{t}(1-\mathrm{X})), \mathrm{q}(\mathrm{t}(1-\mathrm{X})), \mathrm{t}(1-\mathrm{X})\right)}{\partial \mathrm{q}} \\
& -\mathrm{D}_{(\mathrm{y}) \mathrm{X}}^{(\beta, \alpha)}\left(\frac{\partial \mathrm{L}\left(\mathrm{D}_{(\gamma)}^{(\alpha, \beta)} \mathrm{q}(\mathrm{t}(1-\mathrm{X})), \mathrm{q}(\mathrm{t}(1-\mathrm{X})), \mathrm{t}(1-\mathrm{X})\right)}{\partial \mathrm{qX}}\right) 1 \\
& =\left[\begin{array}{l}
{\left[\frac{\mathrm{d} \alpha}{\mathrm{dX}}+\frac{\mathrm{d} \sigma}{\mathrm{dX}}\right] \ln (1-\mathrm{X})} \\
-\frac{\alpha(\mathrm{X})+\sigma(\mathrm{X})-1}{1-\mathrm{X}}-\frac{1}{\mathrm{t}} \psi(\mathrm{t}(1-\mathrm{X}))-\chi \mathrm{t}
\end{array}\right] \\
& \times \frac{\partial \mathrm{L}\left(\mathrm{D}_{(\gamma)}^{(\beta, \alpha)} \mathrm{q}(\mathrm{t}(1-\mathrm{X})), \mathrm{q}(\mathrm{t}(1-\mathrm{X})), \mathrm{t}(1-\mathrm{X})\right)}{\partial \mathrm{q}_{\mathrm{X}}} \\
& +\frac{1}{{ }_{2} \mathrm{~F}_{1}} \frac{\mathrm{d}_{2} \mathrm{~F}_{1}}{\mathrm{dX}} \frac{\partial \mathrm{L}\left(\mathrm{D}_{(\gamma)}^{(\beta, \alpha)} \mathrm{q}(\mathrm{t}(1-\mathrm{X})), \mathrm{q}(\mathrm{t}(1-\mathrm{X})), \mathrm{t}(1-\mathrm{X})\right)}{\partial \mathrm{q}_{\mathrm{X}}}
\end{aligned}
$$

More generally, the two-dimensional generalized FALVA is introduced as follows:

Definition 2: Consider a smooth n-dimensional manifold Mand let $\mathrm{q}: \Omega \subset \mathbb{R}^{2} \rightarrow \mathrm{M}$ be the admissible paths satisfying fixed Dirichlet boundary conditions on $\partial \Omega$. The Lagrangian function $\left(\mathrm{q}_{\mathrm{X}}, \mathrm{q}_{\mathrm{Y}}, \mathrm{q}, \mathrm{t}(1-\mathrm{X})\right), \mathrm{t}(1-$ $\left.\left.\mathrm{Y}))) \rightarrow \mathrm{L}\left(\mathrm{q}_{\mathrm{X}}, \mathrm{q}_{\mathrm{Y}}, \mathrm{q}, \mathrm{t}(1-\mathrm{X})\right), \mathrm{t}(1-\mathrm{Y})\right)\right)$ is supposed to be sufficiently smooth with respect to all its arguments. The two-dimensional generalized FALVA action integral is defined by:

$$
\begin{aligned}
& \left.\left.\mathrm{S}_{(\gamma, \beta, \delta, \xi)}^{(\alpha, \beta]}[\mathrm{q}]=\iint^{{ }^{\Omega}} \mathrm{D}_{(\gamma) ; \mathrm{Y}} \mathrm{L}\left(\mathrm{D}_{(\gamma) ; \mathrm{X}}^{(\alpha, \delta)} \mathrm{q}, \mathrm{q}, \mathrm{t}(1-\mathrm{X})\right), \mathrm{t}(1-\mathrm{Y})\right)\right) \\
& \frac{(1-\mathrm{X})^{\alpha(\mathrm{X})+\sigma(\mathrm{X})-1}}{\Gamma(\alpha(\mathrm{t}(1-\mathrm{X})))} \frac{(1-\mathrm{Y})^{\alpha(\mathrm{Y})+\sigma(\mathrm{Y})-1}}{\Gamma(\alpha(\mathrm{t}(1-\mathrm{Y})))} \\
& \times_{2} F_{1}(a, b ; c ; X){ }_{2} F_{1}(a, b ; c ; Y) e^{\chi \cdot t(1-X)} e^{\sigma \cdot t(1-Y)} d X d Y
\end{aligned}
$$

where, $\mathrm{X} \neq 1$ and $\mathrm{Y} \neq 1 ; \mathrm{q}=(\mathrm{t}(1-\mathrm{X}), \mathrm{t}(1-\mathrm{Y})) ; \mathrm{D}_{(\gamma) \mathrm{x}}^{(\delta, \alpha)}$ and $\mathrm{D}_{(\xi), \mathrm{Y}}^{(\xi, \beta)}$ are the fractional derivative operators (2) respectively of orders $(\delta, \alpha)$ and $(\xi, \beta)$ with respect to $X$ and Y. $\chi, \sigma \in \mathbb{R}$.

Theorem 2: The double-weighed fractional generalized Euler-Lagrange equation associated to the fractional action (17) is:

$$
\begin{aligned}
& \frac{\left.\left.\partial \mathrm{L}\left(\mathrm{D}_{(\gamma) \mathrm{X}}^{(\alpha, \delta)} \mathrm{q}, \mathrm{D}_{(\gamma) ; \mathrm{Y}}^{(\beta, \xi)} \mathrm{q}, \mathrm{q}, \mathrm{t}(1-\mathrm{X})\right), \mathrm{t}(1-\mathrm{Y})\right)\right)}{\partial \mathrm{q}} \\
& -\mathrm{D}_{-(\gamma) \mathrm{X}}^{(\delta, \alpha)}\left(\frac{\left.\left.\partial \mathrm{L}\left(\mathrm{D}_{(\gamma) ; \mathrm{X}}^{(\alpha, \delta)} \mathrm{q}, \mathrm{D}_{(\gamma) ; \mathrm{Y}}^{(\beta, \xi)} \mathrm{q}, \mathrm{q}, \mathrm{t}(1-\mathrm{X})\right), \mathrm{t}(1-\mathrm{Y})\right)\right)}{\partial \mathrm{q}_{\mathrm{X}}}\right) \\
& -\mathrm{D}_{-(\gamma) ; \mathrm{Y}}^{(\xi, \beta)}\left(\frac{\left.\left.\partial \mathrm{L}\left(\mathrm{D}_{(\gamma) \mathrm{X}}^{(\alpha, \delta)} \mathrm{q}, \mathrm{D}_{(\gamma) ; \mathrm{Y}}^{(\beta, \xi)} \mathrm{q}, \mathrm{q}, \mathrm{t}(1-\mathrm{X})\right), \mathrm{t}(1-\mathrm{Y})\right)\right)}{\partial \mathrm{q}_{\mathrm{Y}}}\right)
\end{aligned}
$$

$$
\begin{aligned}
& =\left[\begin{array}{l}
{\left[\frac{\mathrm{d} \alpha}{\mathrm{dX}}+\frac{\mathrm{d} \sigma}{\mathrm{dX}}\right] \ln (1-\mathrm{X})-} \\
\frac{\alpha(X)+\sigma(\mathrm{X})-1}{1-\mathrm{X}}-\frac{1}{\mathrm{t}} \psi(\mathrm{t}(1-\mathrm{X}))-\chi \mathrm{t}
\end{array}\right] \\
& \times \frac{\left.\left.\partial \mathrm{L}\left(\mathrm{D}_{(\gamma) ; \mathrm{X}}^{(\alpha, \delta)} \mathrm{q}, \mathrm{D}_{(\gamma) \mathrm{Y}}^{(\beta, \xi)} \mathrm{q}, \mathrm{q}, \mathrm{t}(1-\mathrm{X})\right), \mathrm{t}(1-\mathrm{Y})\right)\right)}{\partial \mathrm{q}_{\mathrm{X}}}
\end{aligned}
$$

\section{EL-NABULSI Ahmad Rami:}

$$
\begin{aligned}
& +\left[\begin{array}{l}
{\left[\frac{\mathrm{d} \alpha}{\mathrm{dY}}+\frac{\mathrm{d} \sigma}{\mathrm{dY}}\right] \ln (1-\mathrm{Y})-\frac{\alpha(\mathrm{Y})+\sigma(\mathrm{Y})-1}{1-\mathrm{Y}}} \\
-\frac{1}{\mathrm{t}} \psi(\mathrm{t}(1-\mathrm{Y}))-\sigma \mathrm{t}
\end{array}\right] \\
& \times \frac{\left.\left.\partial \mathrm{L}\left(\mathrm{D}_{(\gamma) \mathrm{X}}^{(\alpha, \delta)} \mathrm{q}, \mathrm{D}_{(\gamma) \mathrm{Y}}^{(\beta, \xi)} \mathrm{q}, \mathrm{q}, \mathrm{t}(1-\mathrm{X})\right), \mathrm{t}(1-\mathrm{Y})\right)\right)}{\partial \mathrm{q}_{\mathrm{Y}}} \\
& +\frac{1}{{ }_{2} \mathrm{~F}_{1}} \frac{\mathrm{d}_{2} \mathrm{~F}_{1}}{\mathrm{dX}} \frac{\left.\left.\partial \mathrm{L}\left(\mathrm{D}_{(\gamma) \mathrm{X}}^{(\alpha, \delta)} \mathrm{q}, \mathrm{D}_{(\gamma) ; \mathrm{Y}}^{(\beta, \xi)} \mathrm{q}, \mathrm{q}, \mathrm{t}(1-\mathrm{X})\right), \mathrm{t}(1-\mathrm{Y})\right)\right)}{\partial \mathrm{q}_{\mathrm{X}}} \\
& +\frac{1}{{ }_{2} \mathrm{~F}_{1}} \frac{\mathrm{d}_{2} \mathrm{~F}_{1}}{\mathrm{dY}} \frac{\left.\left.\partial \mathrm{L}\left(\mathrm{D}_{(\gamma) \mathrm{X}}^{(\alpha, \delta)} \mathrm{q}, \mathrm{D}_{(\gamma) ; \mathrm{Y}}^{(\beta, \xi)} \mathrm{q}, \mathrm{q}, \mathrm{t}(1-\mathrm{X})\right), \mathrm{t}(1-\mathrm{Y})\right)\right)}{\partial \mathrm{q}_{\mathrm{Y}}}
\end{aligned}
$$

Proof: We let $\mathrm{w}$ be the minimum solution satisfying the Dirichlet conditions $\mathrm{w}(\mathrm{X}, \mathrm{Y})=0, \forall(\mathrm{X}, \mathrm{Y}) \in \partial \Omega$, h a tiny parameter $\mathrm{h} \in \mathbb{R}$ and $\mathrm{q}$ be the stationary solution so that $\mathrm{q}(\mathrm{t}(1-\mathrm{X}))+\mathrm{hw}(\mathrm{t}(1-\mathrm{X}))$ satisfies the Dirichlet boundary conditions $\forall \mathrm{h} \in \mathbb{R}$. The generalized fractional action is written accordingly like (El-Nabulsi and Torres, 2008):

$$
\begin{aligned}
& \mathrm{S}_{(\gamma)}^{(\alpha, \beta, \delta, \xi)}[\mathrm{q}+\mathrm{hw}]= \\
& \iint_{\Omega} \mathrm{L}\left(\mathrm{D}_{(\gamma) ; \mathrm{x}}^{(\alpha, \delta)} \mathrm{q}+\mathrm{hD} \mathrm{D}_{(\gamma) ; \mathrm{x}}^{(\alpha, \delta)} \mathrm{w},\right. \\
& \left.\left.\left.\iint_{(\gamma) ; Y}^{(\beta, \xi)} q+h D_{(\gamma) ; Y}^{(\beta, \xi)}, q+h w, t(1-X)\right), t(1-Y)\right)\right) \\
& \times \frac{(1-\mathrm{X})^{\alpha(\mathrm{X})+\sigma(\mathrm{X})-1}}{\Gamma(\alpha(\mathrm{t}(1-\mathrm{X})))} \frac{(1-\mathrm{Y})^{\alpha(\mathrm{Y})+\sigma(\mathrm{Y})-1}}{\Gamma(\alpha(\mathrm{t}(1-\mathrm{Y})))} \\
& \times_{2} F_{1}(a, b ; c ; X){ }_{2} F_{1}(a, b ; c ; Y) e^{\chi \cdot t(1-X)} e^{\sigma \cdot t(1-Y)} d X d Y
\end{aligned}
$$

Therefore:

$$
\begin{aligned}
& \left.\frac{\mathrm{d}}{\mathrm{dh}} \mathrm{S}_{(\gamma)}^{(\alpha, \beta, \delta, \xi)}[\mathrm{q}+\mathrm{hw}]\right|_{\mathrm{h}=0}=\iint_{\Omega}\left(\begin{array}{l}
\mathrm{w} \frac{\partial \mathrm{L}}{\partial \mathrm{q}}+\mathrm{D}_{(\gamma) ; \mathrm{X}}^{(\alpha, \delta)} \\
\mathrm{w} \frac{\partial \mathrm{L}}{\partial \mathrm{q}_{\mathrm{X}}}+\mathrm{D}_{(\gamma) ; \mathrm{Y}}^{(\beta, \xi)} \mathrm{w} \frac{\partial \mathrm{L}}{\partial \mathrm{q}_{\mathrm{Y}}}
\end{array}\right) \\
& \times \frac{(1-\mathrm{X})^{\alpha(\mathrm{X})+\sigma(\mathrm{X})-1}}{\Gamma(\alpha(\mathrm{t}(1-\mathrm{X})))} \frac{(1-\mathrm{Y})^{\alpha(\mathrm{Y})+\sigma(\mathrm{Y})-1}}{\Gamma(\alpha(\mathrm{t}(1-\mathrm{Y})))} \\
& \times{ }_{2} \mathrm{~F}_{1}(\mathrm{a}, \mathrm{b} ; \mathrm{c} ; \mathrm{X})_{2} \mathrm{~F}_{1}(\mathrm{a}, \mathrm{b} ; \mathrm{c} ; \mathrm{Y}) \mathrm{e}^{\chi \cdot \mathrm{t}(1-\mathrm{X})} \mathrm{e}^{\sigma \cdot t(1-\mathrm{Y})} \mathrm{dXdY}
\end{aligned}
$$

Integration by parts and making use of the Green's theorems: 


$$
\begin{aligned}
& \iint_{\Omega \subset \mathbb{R}^{2}}\left(\frac{\partial \mathrm{P}}{\partial \xi} \mathrm{G}_{1}+\frac{\partial \mathrm{P}}{\partial \lambda} \mathrm{G}_{2}\right) \mathrm{d} \bar{\xi} \mathrm{d} \bar{\lambda}=\oint_{\partial \Omega} \\
& \mathrm{P}\left(-\mathrm{G}_{2} \mathrm{~d} \bar{\xi}+\mathrm{G}_{1} \mathrm{~d} \bar{\lambda}\right)-\iint_{\Omega \subset \mathbb{R}^{2}}\left(\mathrm{P}\left(\frac{\partial \mathrm{G}_{1}}{\partial \xi}+\frac{\partial \mathrm{G}_{2}}{\partial \lambda}\right)\right) \mathrm{d} \bar{\xi} \mathrm{d} \bar{\lambda}
\end{aligned}
$$

Where:

$$
\begin{aligned}
& \mathrm{d} \bar{\xi}=\frac{(1-\mathrm{X})^{\alpha(\mathrm{X})+\sigma(\mathrm{X})-1}}{\Gamma(\alpha(\mathrm{t}(1-\mathrm{X})))}{ }_{2} \mathrm{~F}_{1}(\mathrm{a}, \mathrm{b} ; \mathrm{c} ; \mathrm{X}) \mathrm{e}^{\chi \cdot \mathrm{t}(1-\mathrm{X})} \mathrm{dX} \\
& \mathrm{d} \bar{\lambda}=\frac{(1-\mathrm{Y})^{\alpha(\mathrm{Y})+\sigma(\mathrm{Y})-1}}{\Gamma(\alpha(\mathrm{t}(1-\mathrm{Y})))}{ }_{2} \mathrm{~F}_{1}(\mathrm{a}, \mathrm{b} ; \mathrm{c} ; \mathrm{Y}) \mathrm{e}^{\sigma \cdot \mathrm{t}(1-\mathrm{Y})} \mathrm{dY}
\end{aligned}
$$

we find for any smooth functions $G_{1}$ and $G_{2}$ :

$$
\begin{aligned}
& \left.\frac{\mathrm{d}}{\mathrm{dh}} \mathrm{S}_{(\gamma)}^{(\alpha, \beta, \delta, \xi)}[\mathrm{q}+\mathrm{hw}]\right|_{\mathrm{h}=0}: \\
& -\iint_{\Omega} \mathrm{w}\left[\left(\mathrm{D}_{(\gamma) \mathrm{X}}^{(\alpha, \delta)}\left(\frac{\left.\left.\partial \mathrm{L}\left(\mathrm{D}_{(\gamma, \mathrm{X}) \mathrm{X}}^{(\alpha, \delta)} \mathrm{q}, \mathrm{D}_{(\gamma) \mathrm{Y}}^{(\beta, \xi)} \mathrm{q}, \mathrm{q}, \mathrm{t}(1-\mathrm{X})\right), \mathrm{t}(1-\mathrm{Y})\right)\right)}{\partial \mathrm{q}_{\mathrm{X}}}\right)\right.\right.
\end{aligned}
$$

\section{EL-NABULSI Ahmad Rami:}

$$
\begin{aligned}
& \left.+\mathrm{D}_{(\gamma) \mathrm{Y}}^{(\beta, \xi)}\left(\frac{\left.\left.\partial \mathrm{L}\left(\mathrm{D}_{(\gamma) \mathrm{X}}^{(\alpha, \delta)} \mathrm{q}, \mathrm{D}_{(\gamma): \mathrm{Y}}^{(\beta, \mathrm{s})} \mathrm{q}, \mathrm{q}, \mathrm{t}(1-\mathrm{X})\right), \mathrm{t}(1-\mathrm{Y})\right)\right)}{\partial \mathrm{q}_{\mathrm{Y}}}\right)\right] \\
& +\left\{\left[\left[\frac{\mathrm{d} \alpha}{\mathrm{dX}}+\frac{\mathrm{d} \sigma}{\mathrm{dX}}\right] \ln (1-\mathrm{X})-\frac{\alpha(\mathrm{X})+\sigma(\mathrm{X})-1}{1-\mathrm{X}}-\frac{1}{\mathrm{t}} \psi(\mathrm{t}(1-\mathrm{X}))-\chi \mathrm{t}\right]\right. \\
& \times \frac{\left.\left.\partial \mathrm{L}\left(\mathrm{D}_{(\gamma) ;}^{(\alpha, \delta)} \mathrm{q}, \mathrm{D}_{(\gamma) ;}^{(\beta, \xi)} \mathrm{q}, \mathrm{q}, \mathrm{t}(1-\mathrm{X})\right), \mathrm{t}(1-\mathrm{Y})\right)\right)}{\partial \mathrm{q}_{\mathrm{X}}} \\
& +\left[\left[\frac{\mathrm{d} \alpha}{\mathrm{dY}}+\frac{\mathrm{d} \sigma}{\mathrm{dY}}\right] \ln (1-\mathrm{Y})-\frac{\alpha(\mathrm{Y})+\sigma(\mathrm{Y})-1}{1-\mathrm{Y}}-\frac{1}{\mathrm{t}} \psi(\mathrm{t}(1-\mathrm{Y}))-\sigma \mathrm{t}\right] \\
& \times \frac{\left.\left.\partial \mathrm{L}\left(\mathrm{D}_{(\gamma): \mathrm{X}}^{(\alpha, \delta)} \mathrm{q}, \mathrm{D}_{(\gamma) ; \mathrm{Y}}^{(\beta, \xi)} \mathrm{q}, \mathrm{q}, \mathrm{t}(1-\mathrm{X})\right), \mathrm{t}(1-\mathrm{Y})\right)\right)}{\partial \mathrm{q}_{\mathrm{Y}}} \\
& +\frac{1}{{ }_{2} \mathrm{~F}_{1}} \frac{\mathrm{d}_{2} \mathrm{~F}_{1}}{\mathrm{dX}} \frac{\left.\left.\partial \mathrm{L}\left(\mathrm{D}_{(\gamma) ; \mathrm{X}}^{(\alpha, \delta)} \mathrm{q}, \mathrm{D}_{(\gamma) \mathrm{Y}}^{(\beta, \mathrm{Y})} \mathrm{q}, \mathrm{q}, \mathrm{t}(1-\mathrm{X})\right), \mathrm{t}(1-\mathrm{Y})\right)\right)}{\partial \mathrm{q}_{\mathrm{X}}} \\
& +\frac{1}{{ }_{2} \mathrm{~F}_{1}} \frac{\mathrm{d}_{2} \mathrm{~F}_{1}}{\mathrm{dY}} \frac{\left.\left.\partial \mathrm{L}\left(\mathrm{D}_{(\gamma) ; \mathrm{X}}^{(\alpha, \delta)} \mathrm{q}, \mathrm{D}_{(\gamma) ; \mathrm{Y}}^{(\beta, \xi)} \mathrm{q}, \mathrm{q}, \mathrm{t}(1-\mathrm{X})\right), \mathrm{t}(1-\mathrm{Y})\right)\right)}{\partial \mathrm{q}_{\mathrm{Y}}} \\
& \left.-\frac{\left.\left.\partial \mathrm{L}\left(\mathrm{D}_{(\gamma): \mathrm{X}}^{(\alpha, \delta)} \mathrm{q}, \mathrm{D}_{(\gamma) \mathrm{Y}}^{(\beta, \xi)} \mathrm{q}, \mathrm{q}, \mathrm{t}(1-\mathrm{X})\right), \mathrm{t}(1-\mathrm{Y})\right)\right)}{\partial \mathrm{q}}\right\} \mathrm{dXdY}
\end{aligned}
$$

Because of the arbitrariness of $\mathrm{w}(\mathrm{X}, \mathrm{Y})$ inside the domain $\Omega$, it follows the generalized fractional EulerLagrange equations.

All the previous arguments can be repeated, mutatis mutandis, to the N-dimensional problem. We assume as previously, that the admissible paths are smooth functions $\mathrm{q}: \Omega \subset \mathbb{R}^{\mathrm{N}} \rightarrow \mathrm{M}$ satisfying given Dirichlet boundary conditions on $\partial \Omega$ (El-Nabulsi and Torres, 2008).

Definition 3: The Lagrangian $\left(\mathrm{q}_{\mathrm{X}_{1}}, \ldots, \mathrm{q}_{\mathrm{X}_{\mathrm{N}}}, \mathrm{q}, \mathrm{X}_{1}, \ldots, \mathrm{X}_{\mathrm{N}}\right) \rightarrow \mathrm{L}\left(\mathrm{q}_{\mathrm{X}_{1}}, \ldots, \mathrm{q}_{\mathrm{X}_{\mathrm{N}}}, \mathrm{q}, \mathrm{X}_{1}, \ldots, \mathrm{X}_{\mathrm{N}}\right)$ is to be sufficiently smooth with respect to all its arguments. The N-dimensional fractional generalized-FALVA action integral is defined by:

$$
\begin{aligned}
& \mathrm{S}_{(x)}^{(\alpha, \beta)}[\mathrm{q}]=\int \ldots \int_{\Omega(\xi)} \mathrm{L}\left(\nabla_{\gamma}^{\alpha, \delta} \mathrm{q}(\mathrm{t}(1-\mathrm{X})), \mathrm{q}(\mathrm{t}(1-\mathrm{X})), \mathrm{t}(1-\mathrm{X})\right) \\
& \times \prod_{\mathrm{i}=1}^{\mathrm{N}} \frac{\left(1-\mathrm{X}_{\mathrm{i}}\right)^{\alpha\left(\mathrm{X}_{\mathrm{i}}\right)+\sigma\left(\mathrm{X}_{\mathrm{i}}\right)-1}}{\Gamma\left(\alpha_{\mathrm{i}}\left(\mathrm{t}\left(1-\mathrm{X}_{\mathrm{i}}\right)\right)\right)}{ }_{2} \mathrm{~F}_{1}\left(\mathrm{a}, \mathrm{b} ; \mathrm{c} ; \mathrm{X}_{\mathrm{i}}\right) \mathrm{e}^{\mathrm{X}_{\mathrm{i}} \cdot \mathrm{t}\left(1-\mathrm{X}_{\mathrm{i}}\right)} \mathrm{dX}
\end{aligned}
$$

where, $\nabla_{\gamma}^{\alpha, \delta}=\left(D_{\gamma ; X_{1}}^{\alpha_{1}, \delta_{1}}, \ldots, D_{\gamma ; X_{N}}^{\alpha_{N}, \delta_{N}}\right)$ and $d x=\mathrm{dx}_{1} \ldots \mathrm{dx}_{\mathrm{N}}$.

Theorem 3: The N-dimensional associated fractional Euler-Lagrange equation is given by:

$$
\sum_{i=1}^{N}\left[D_{-\gamma ; X_{i}}^{\alpha_{i}, \delta_{i}}\left(\frac{\partial L}{\partial q_{X_{i}}}\right)+\left[\begin{array}{l}
{\left[\frac{d \alpha}{d X_{i}}+\frac{d \sigma}{d X_{i}}\right] \ln \left(1-X_{i}\right)-} \\
\frac{\alpha\left(X_{i}\right)+\sigma\left(X_{i}\right)-1}{1-X_{i}}- \\
\frac{1}{t} \psi\left(t\left(1-X_{i}\right)\right)-\chi_{i} t
\end{array}\right] \frac{\partial L}{\partial q_{X_{i}}}\right.
$$

\section{EL-NABULSI Ahmad Rami:}

$$
\left.+\frac{1}{{ }_{2} \mathrm{~F}_{1}} \frac{\mathrm{d}_{2} \mathrm{~F}_{1}}{\mathrm{dX}_{\mathrm{i}}} \frac{\partial \mathrm{L}}{\partial \mathrm{q}_{\mathrm{X}_{\mathrm{i}}}}\right]-\frac{\partial \mathrm{L}}{\partial \mathrm{q}}=0
$$

where all partial derivatives of the Lagrangian are evaluated at $\left(\nabla_{\gamma}^{\alpha, \delta} \mathrm{q}(\mathrm{t}(1-\mathrm{X})), \mathrm{q}(\mathrm{t}(1-\mathrm{X})), \mathrm{t}(1-\mathrm{X})\right), \mathrm{X} \in \Omega$.

Generalized lagrangian dynamics: Generalized Lagrangian dynamics may be obtained if we assume that the dynamical fractional exponent is complex, i.e.:

$$
\alpha(\mathrm{X})=\mathrm{F}(\mathrm{X})+\mathrm{iG}(\mathrm{X}) \in \mathbb{C}
$$

and:

$\sigma(\mathrm{X})=\mathrm{E}(\mathrm{X})+\mathrm{iD}(\mathrm{X}) \in \mathbb{C}$

$\mathrm{D}(\mathrm{X}), \mathrm{E}(\mathrm{X}), \mathrm{F}(\mathrm{X}), \mathrm{G}(\mathrm{X})$ are any real function of $\mathrm{x}$. Consequently, Eq. 20 is entitled "complex fractional order generalization" of the Euler-Lagrange equation. For this, we may perform the dependent variable complex and write: 
$\mathrm{q}(\mathrm{t}(1-\mathrm{X}))=\mathrm{q}_{1}(\mathrm{t}(1-\mathrm{X}))+\mathrm{iq}_{2}(\mathrm{t}(1-\mathrm{X}))$

We may use naturally the following equations:

$\frac{\partial}{\partial \mathrm{q}}=\frac{1}{2}\left(\frac{\partial}{\partial \mathrm{q}_{1}}-\mathrm{i} \frac{\partial}{\partial \mathrm{q}_{2}}\right)$

$\frac{\partial}{\partial \mathrm{q}_{\mathrm{x}_{\mathrm{i}}}}=\frac{1}{2}\left(\frac{\partial}{\partial \mathrm{q}_{1 \mathrm{X}_{\mathrm{i}}}}-\mathrm{i} \frac{\partial}{\partial \mathrm{q}_{2 \mathrm{X}_{\mathrm{i}}}}\right)$

$\mathrm{D}_{-\gamma ; \mathrm{X}_{\mathrm{i}}}^{\alpha_{\mathrm{i}}, \delta_{\mathrm{i}}}=\frac{1}{2}\left[\mathrm{D}_{\left(\mathrm{a}_{+}\right) ; \mathrm{X}_{\mathrm{i}}}^{\left(\alpha_{\mathrm{i}}\right)}-\mathrm{D}_{\left(\mathrm{b}_{-}\right) ; \mathrm{X}_{\mathrm{i}}}^{\left(\beta_{\mathrm{i}}\right)}\right]$

$-\frac{i \gamma}{2}\left[D_{\left(a_{+}\right) ; x_{i}}^{\left(\alpha_{i}\right)}+D_{\left(b_{-}\right) ; x_{i}}^{\left(\beta_{i}\right)}\right] \equiv D_{-\gamma ; x_{i}}^{\alpha_{i}, \delta_{i} ;+}-i \gamma D_{-\gamma ; X_{i}}^{\alpha_{i}, \delta_{i} ;-}$

Where:

$\mathrm{D}_{-\gamma ; \mathrm{X}_{\mathrm{i}}}^{\alpha_{\mathrm{i}}, \delta_{i} ;+} \triangleq \frac{1}{2}\left[\mathrm{D}_{\left(\mathrm{a}_{+}\right) ; \mathrm{X}_{\mathrm{i}}}^{\left(\alpha_{\mathrm{i}}\right)}-\mathrm{D}_{\left(\mathrm{b}_{-}\right) ; \mathrm{X}_{\mathrm{i}}}^{\left(\beta_{\mathrm{i}}\right)}\right]$

$\mathrm{D}_{-\gamma ; \mathrm{X}_{\mathrm{i}}}^{\alpha_{\mathrm{i}}, \delta_{i} ;-} \triangleq \frac{1}{2}\left[\mathrm{D}_{\left(\mathrm{a}_{+}\right) ; \mathrm{X}_{\mathrm{i}}}^{\left(\alpha_{\mathrm{i}}\right)}+\mathrm{D}_{\left(\mathrm{b}_{-}\right) ; \mathrm{X}_{\mathrm{i}}}^{\left(\beta_{\mathrm{i}}\right)}\right]$

Choosing $\gamma=\mathrm{a}+\mathrm{ib} \in \mathbb{C},(\mathrm{a}, \mathrm{b}) \in \mathbb{R}$, we may write Eq. 23 like:

$\mathrm{D}_{-\gamma ; \mathrm{X}_{\mathrm{i}}}^{\alpha_{\mathrm{i}}, \delta_{\mathrm{i}}}=\mathrm{D}_{-\gamma ; \mathrm{X}_{\mathrm{i}}}^{\alpha_{\mathrm{i}}, \delta_{\mathrm{i}} ;+}+\mathrm{bD}_{-\gamma ; \mathrm{X}_{\mathrm{i}}}^{\alpha_{\mathrm{i}}, \delta_{\mathrm{i}} ;-}-\mathrm{iaD} \mathrm{D}_{-\gamma ; \mathrm{X}_{\mathrm{i}}}^{\alpha_{\mathrm{i}}, \delta_{\mathrm{i}} ;-} \equiv \overline{\mathrm{D}}_{-\gamma ; \mathrm{x}_{\mathrm{i}}}^{\alpha_{\mathrm{i}}, \delta_{\mathrm{i}} ;+}-\underline{\mathrm{i}}_{-\gamma ; \mathrm{X}_{\mathrm{i}}}^{\alpha_{\mathrm{i}}, \delta_{\mathrm{i}} ;+}$

Where:

$\overline{\mathrm{D}}_{-\gamma ; \mathrm{X}_{\mathrm{i}}}^{\alpha_{\mathrm{i}}, \delta_{\mathrm{i}} ;+}=\mathrm{D}_{-\gamma ; \mathrm{X}_{\mathrm{i}}}^{\alpha_{\mathrm{i}}, \delta_{i} ;+}+\mathrm{bD}_{-\gamma ; \mathrm{X}_{\mathrm{i}}}^{\alpha_{\mathrm{i}}, \delta_{\mathrm{i}} ;-}=\mathfrak{R} \mathrm{D}_{-\gamma ; \mathrm{X}_{\mathrm{i}}}^{\alpha_{\mathrm{i}}, \delta_{\mathrm{i}} ;+}$

$\underline{\mathrm{D}}_{-\gamma ; \mathrm{x}_{\mathrm{i}}}^{\alpha_{\mathrm{i}}, \delta_{\mathrm{i}} ;+}=\mathrm{aD}_{-\gamma ; \mathrm{X}_{\mathrm{i}}}^{\alpha_{\mathrm{i}}, \delta_{\mathrm{i}} ;-}=\mathfrak{J} \mathrm{D}_{-\gamma ; \mathrm{x}_{\mathrm{i}}}^{\alpha_{\mathrm{i}}, \delta_{\mathrm{i}}}$

and accordingly, Eq. 20 is rewritten like.

\section{EL-NABULSI Ahmad Rami:}

$$
\begin{aligned}
& \sum_{\mathrm{i}=1}^{\mathrm{N}}\left[\left(\overline{\mathrm{D}}_{-\gamma ; \mathrm{X}_{\mathrm{i}}}^{\alpha_{\mathrm{i}}, \mathrm{S}_{i} ;+}-\underline{\mathrm{D}}_{-\gamma ; \mathrm{X}_{\mathrm{i}}}^{\alpha_{\mathrm{i}}, \delta_{i} ;+}\right)\left(\frac{1}{2}\left(\frac{\partial \mathrm{L}}{\partial \mathrm{q}_{1 \mathrm{X}_{\mathrm{i}}}}-\mathrm{i} \frac{\partial \mathrm{L}}{\partial \mathrm{q}_{2 \mathrm{X}_{\mathrm{i}}}}\right)\right)+\right. \\
& \frac{1}{2}\left[\left[\frac{\mathrm{dF}}{\mathrm{d} \mathrm{X}_{\mathrm{i}}}+\frac{\mathrm{dE}}{\mathrm{d} \mathrm{X}_{\mathrm{i}}}+\mathrm{i}\left(\frac{\mathrm{dG}}{\mathrm{dX}}+\frac{\mathrm{dD}}{\mathrm{dX}}\right)\right] \ln \left(1-\mathrm{X}_{\mathrm{i}}\right)\right. \\
& \left.-\frac{\mathrm{E}(\mathrm{X})+\mathrm{F}(\mathrm{X})+\mathrm{i}(\mathrm{D}(\mathrm{X})+\mathrm{G}(\mathrm{X}))-1}{1-\mathrm{X}_{\mathrm{i}}}\right]\left(\frac{\partial \mathrm{L}}{\partial \mathrm{q}_{1 \mathrm{X}_{\mathrm{i}}}}-\mathrm{i} \frac{\partial \mathrm{L}}{\partial \mathrm{q}_{2 \mathrm{X}_{\mathrm{i}}}}\right) \\
& -\frac{1}{\mathrm{t}} \psi\left(\mathrm{t}\left(1-\mathrm{X}_{\mathrm{i}}\right)\right)-\chi_{\mathrm{i}} \mathrm{t} \\
& +\frac{1}{2} \frac{1}{{ }_{2} \mathrm{~F}_{1}} \frac{\mathrm{d}_{2} \mathrm{~F}_{1}}{\mathrm{dX}_{\mathrm{i}}}\left(\frac{\partial \mathrm{L}}{\partial \mathrm{q}_{1 \mathrm{X}_{\mathrm{i}}}}-\mathrm{i} \frac{\partial \mathrm{L}}{\partial \mathrm{q}_{2 \mathrm{X}_{\mathrm{i}}}}\right)-\frac{1}{2}\left(\frac{\partial \mathrm{L}}{\partial \mathrm{q}_{1}}-\mathrm{i} \frac{\partial \mathrm{L}}{\partial \mathrm{q}_{2}}\right)=0
\end{aligned}
$$

which in its turn may be splitted into the equations by separating the real from the imaginary parts:

$$
\begin{aligned}
& \sum_{\mathrm{i}=1}^{\mathrm{N}}\left[\left(\overline{\mathrm{D}}_{-\gamma ; \mathrm{X}_{\mathrm{i}}}^{\alpha_{i}, \delta_{i} ;+}\left(\frac{\partial \mathrm{L}}{\partial \mathrm{q}_{1 \mathrm{X}_{\mathrm{i}}}}\right)-\underline{\mathrm{D}}_{-\gamma ; \mathrm{X}_{\mathrm{i}}}^{\alpha_{i}, \delta_{i} ;+}\left(\frac{\partial \mathrm{L}}{\partial \mathrm{q}_{2 \mathrm{X}_{\mathrm{i}}}}\right)\right)\right. \\
& +\frac{1}{2}\left[\left[\frac{\mathrm{dF}}{\mathrm{d} \mathrm{X}_{\mathrm{i}}}+\frac{\mathrm{dE}}{\mathrm{d} \mathrm{X}_{\mathrm{i}}}\right] \ln \left(1-\mathrm{X}_{\mathrm{i}}\right)\right. \\
& \left.-\frac{\mathrm{E}(\mathrm{X})+\mathrm{F}(\mathrm{X})-1}{1-\mathrm{X}_{\mathrm{i}}}-\frac{1}{\mathrm{t}} \psi\left(\mathrm{t}\left(1-\mathrm{X}_{\mathrm{i}}\right)\right)-\chi_{\mathrm{i}} \mathrm{t}\right]\left(\frac{\partial \mathrm{L}}{\partial \mathrm{q}_{1 \mathrm{X}_{\mathrm{i}}}}\right) \\
& +\frac{1}{2}\left[\left(\frac{\mathrm{dG}}{\mathrm{d} \mathrm{X}_{\mathrm{i}}}+\frac{\mathrm{dD}}{\mathrm{dX}}\right) \ln \left(1-\mathrm{X}_{\mathrm{i}}\right)-\frac{(\mathrm{D}(\mathrm{X})+\mathrm{G}(\mathrm{X}))}{1-\mathrm{X}_{\mathrm{i}}}\right] \\
& \left.\left(\frac{\partial \mathrm{L}}{\partial \mathrm{q}_{2 \mathrm{X}_{\mathrm{i}}}}\right)+\frac{1}{{ }_{2} \mathrm{~F}_{1}} \frac{\mathrm{d}_{2} \mathrm{~F}_{1}}{\mathrm{dX}_{\mathrm{i}}}\left(\frac{\partial \mathrm{L}}{\partial \mathrm{q}_{1 \mathrm{X}_{\mathrm{i}}}}\right)\right]-\left(\frac{\partial \mathrm{L}}{\partial \mathrm{q}_{1}}\right)=0
\end{aligned}
$$

and:

$$
\begin{aligned}
& \sum_{\mathrm{i}=1}^{\mathrm{N}}\left[-\overline{\mathrm{D}}_{-\gamma ; \mathrm{X}_{\mathrm{i}} \alpha_{\mathrm{i}}, \delta_{i} ;+}\left(\frac{\partial \mathrm{L}}{\partial \mathrm{q}_{2 \mathrm{X}_{\mathrm{i}}}}\right)-\underline{\mathrm{D}}_{-\gamma ; \mathrm{X}_{\mathrm{i}}}^{\alpha_{\mathrm{i}}, \delta_{i} ;+}\left(\frac{\partial \mathrm{L}}{\partial \mathrm{q}_{1 \mathrm{X}_{\mathrm{i}}}}\right)\right. \\
& -\left[\left[\frac{\mathrm{dF}}{\mathrm{d} \mathrm{X}_{\mathrm{i}}}+\frac{\mathrm{dE}}{\mathrm{d} \mathrm{X}_{\mathrm{i}}}\right] \ln \left(1-\mathrm{X}_{\mathrm{i}}\right)\right. \\
& \left.-\frac{\mathrm{E}(\mathrm{X})+\mathrm{F}(\mathrm{X})}{1-\mathrm{X}_{\mathrm{i}}}-\frac{1}{\mathrm{t}} \psi\left(\mathrm{t}\left(1-\mathrm{X}_{\mathrm{i}}\right)\right)-\chi_{\mathrm{i}} \mathrm{t}\right]\left(\frac{\partial \mathrm{L}}{\partial \mathrm{q}_{2 \mathrm{X}_{\mathrm{i}}}}\right) \\
& +\left[\frac{1}{2}\left(\frac{\mathrm{dG}}{\mathrm{d} \mathrm{X}_{\mathrm{i}}}+\frac{\mathrm{dD}}{\mathrm{d} \mathrm{X}_{\mathrm{i}}}\right) \ln \left(1-\mathrm{X}_{\mathrm{i}}\right)-\mathrm{i} \frac{(\mathrm{D}(\mathrm{X})+\mathrm{G}(\mathrm{X}))}{1-\mathrm{X}_{\mathrm{i}}}\right] \\
& \left.\left(\frac{\partial \mathrm{L}}{\partial \mathrm{q}_{1 \mathrm{X}_{\mathrm{i}}}}\right)-\frac{1}{{ }_{2} \mathrm{~F}_{1}} \frac{\mathrm{d}_{2} \mathrm{~F}_{1}}{\mathrm{dX}}\left(\frac{\partial \mathrm{L}}{\partial \mathrm{q}_{2 \mathrm{X}_{\mathrm{i}}}}\right)\right]+\left(\frac{\partial \mathrm{L}}{\partial \mathrm{q}_{2}}\right)=0
\end{aligned}
$$

These equations require numerical solutions and it is the author's speculation that they may play important role on complexified oscillatory dynamical systems with time-dependent mass and time-dependent frequency. Work in this direction is also under progress. Naturally, one expects a complex generalization of Hamiltonian dynamics which certainly will guides to a new dynamics which may differ completely from the classical mechanics cardinally and may bring interesting consequences, e.g. quantum field theory, control theory and hence we expect that exotic solutions will be physical.

\section{MATERIALS AND METHODS}

Recently, a lot of attention has been put on the fractional calculus of variations. In this work we use the concept of Saxena-Kumbhat fractional integral to 
explore some new aspects of quantum field theory on multifractal sets. The method is based on the concept of Fractional ActionLike Variational Approach recently introduced by the author. We have laid out the groundwork for generalizing the non-conservative dissipative systems using the methodology of the Saxena-Kumbhat hypergeometric fractional integral within the framework of the fractional action-like variational approach by introducing the basic settings.

\section{RESULTS AND DISCUSSION}

An important additional feature of the new formalism is the hierarchy of differential equations that can be obtained from the generalized fractional EulerLagrange equations. Besides, the resulting fractional Lagrangian and Hamiltonian dynamics may be complexified. This fact may have interesting consequences in different aspects of quantum dynamical Hamiltonian systems having discrete spectra and exhibiting the PT symmetry, i.e., dynamical equations invariant under combined space and time. To the best of our knowledge, the question of obtaining conditions on the Lagrangian assuring the existence of stationary trajectories is an entirely open question in the fractional scenery. An important additional feature of the new formalism is the hierarchy of differential equations that can be obtained from the generalized fractional Euler-Lagrange equations. Future research efforts may be directed towards formulating predictions that can be tested numerically. Furthermore, complex trajectories of quantum mechanical systems must be addressed and work along these lines is presently underway.

\section{CONCLUSION}

Contemporaneous research efforts are required to validate or falsify, develop or disprove the fractional dynamics discussed here including our preliminary findings.

\section{ACKNOWLEDGMENT}

I would like to thanks Professor Manuel Duarte Ortigueira for inviting me to participate on the Symposium on Fractional Signals and Systems which will be held at Lisbon-Portugal.

\section{REFERENCES}

Aghaei, M.R.S., Z.A. Zukarnain, A. Mamat and H. Zainuddin, 2009. A hybrid architecture approach for quantum algorithms. J. Comput. Sci., 5: 725-731. DOI: 10.3844/jessp.2009.725.731
Al-Daoud, E., 2008. A new quantum key distribution protocol. Am. J. Applied Sci., 5: 93-96. DOI: 10.3844/ajassp.2008.93.96

Ali, S., S. Saharudin and M.R.B. Wahiddin, 2009. Quantum key distribution using decoy state protocol. Am. J. Eng. Applied Sci., 2: 694-698. DOI: 10.3844/ajeassp.2009.694.698

Bitev, V.V., M.Y. Kalmikov and B.A. Kniehl, 2009. Differential reduction of generalized hypergeometric functions in application to Feynman diagrams: One-variable case. http://www.mendeley.com/research/differentialreduction-of-generalized-hypergeometricfunctions-in-application-to-feynman-diagramsonevariable-case/

Cresson, J., 2007. Fractional embedding of differential operators and Lagrangian systems. J. Math. Phys. 48: 34. DOI: $10.1063 / 1.2483292$

El-Nabulsi, R.A. and D.F.M. Torres, 2007. Necessary optimality condition for fractional action-like variational approach with-Liouville derivatives of order $(\alpha, \beta)$, Math. Meth. Appl. Sci. 30: 1931-1939.

El-Nabulsi, R.A. and D.F.M. Torres, 2008. Fractional actionlike variational problems, J. Math. Phys. 49, 053521-053527. DOI: 10.1063/1.2929662

El-Nabulsi, R.A., 2005a. A fractional approach to nonconservative lagrangian dynamical systems, Fizika A, 14: 289-298.

El-Nabulsi, R.A., 2005b. A fractional action-like variational approach of some classical, quantum and geometrical dynamics, Int. J. Appl. Math., 17: 299-317.

El-Nabulsi, R.A., 2007. Geometry of manifolds on Lie endomorphism space and their duals under fractional action-like variational approach, Fizika A, 16: 137-156.

El-Nabulsi, R.A., 2008a. Fractional field theories from multi-dimensional fractional variational problems, Int. J. Mod. Geom. Meth. Mod. Phys. 5: 863. DOI: 10.1142/S0219887808003119

El-Nabulsi, R.A., 2008b. Black hole growth and accretion energy from fractional action-like variational approach. Fizika B17, 3: 369-378.

El-Nabulsi, R.A., 2008c. Subdiffusion over fractional quantum paths without fractional derivative, Fizika A, 17: 71.

El-Nabulsi, R.A., 2009a. Fractional quantum EulerCauchy equation in the Schrödinger picture, complexified harmonic oscillators and emergence of complexified lagrangian and hamiltonian dynamics. Mod. Phys. Lett. B, 23: 3369-3386. DOI: $10.1142 / \mathrm{S} 0217984909021387$ 
El-Nabulsi, R.A., 2009b. Fractional action-like variational problems in holonomic, non-holonomic and semi-holonomic constrained and dissipative dynamical systems, Chaos, Solitons and Fractals 42 (2009) 52

El-Nabulsi, R.A., 2009c. On the fractional minimal length Heisenberg-Weyl uncertainty relation from fractional Riccati generalized momentum operator, Chaos, Solitons Fractals, 42: 84-88. DOI: 10.1016/j.chaos.2008.10.031

El-Nabulsi, R.A., 2009d. Fractional dynamics, fractional weak bosons masses and physics beyond the standard model. Chaos, Solitons Fractals, 41: 2262-2270. DOI: 10.1016/j.chaos.2008.08.033

El-Nabulsi, R.A., 2009e. Fractional Dirac operators and deformed field theory on Clifford algebra. Chaos, Solitons Fractals, 42: 2614-2622. DOI: 10.1016/j.chaos.2009.04.002

El-Nabulsi, R.A., 2009f. Complexified quantum field theory and "mass without mass" from multidimensional fractional actionlike variational approach with dynamical fractional exponent. Chaos, Solitons Fractals, 42: 2384-2398. DOI: 10.1016/j.chaos.2009.03.115

El-Nabulsi, R.A., 2009g. Fractional illusion theory of space: fractional gravitational field with fractional extra-dimensions, Chaos, Solitons Fractals, 42: 377-384. DOI: 10.1016/j.chaos.2008.12.008

Gomes, D. and E.C.D. Oliveira, 2004. The secondorder Klein-Gordon field equation. Int. J. Math. Math. Sci., 69: 3775-3781. DOI: $10.1155 / \mathrm{S} 0161171204406565$

Granik, A., 2004. On a loss of information in a transition from quantum to a quasi-classical regime. Am. J. Applied Sci., 1: 255-257. DOI: 10.3844/ajassp.2004.255.257

Hilfer, R., 2000. Applications of Fractional Calculus in Physics. 1st Edn., Word Scientific Publishing Co., New Jersey, London, ISBN-10: 9810234570, pp: 463.

Kluwer, D., 2004. Fractional derivatives and their applications. 38: 1-489.
Kobolev, L.Y., 2000. What dimensions do the time and space have: integer or fractional. http://arxiv.org/abs/physics/0001035

Michel, N. and M.V. Soitsov, 2008. Fast computation of the Gauss hypergeometric function with all its entire parameters complex with application to the Poschl-Teller-Ginocchio potential wave functions. Comput. Phy. Communi., 178: 535-551.

Miller, K.S. and B. Ross, 1993. An Introduction to the Fractional Calculus and Fractional Differential Equations. 1st Edn., John Wiley and Sons Inc., New York, ISBN-10: 0471588849, pp: 366.

Ortigueira, M.D. and J.A.T. Machado, 2006. Fractional calculus applications in signals and systems. Signal Processing-Fractional Calculus Applications Signals Syst., 86: 2503-2504. DOI: 10.1016/j.sigpro.2006.02.001

Ortigueira, M.D. and J.A.T. Machado, 2008. Special Issue on "Discontinuous and Fractional Dynamical Systems". ASME J. Comput. Nonlinear Dynamics, 3: 1. DOI: $10.1115 / 1.2834905$

Podlubny, I., 1999. Fractional Differential Equations: An Introduction to Fractional Derivatives, Fractional Differential Equations, to Methods of their Solution and some of their Applications. 1st Edn., Academic Press, New York-London, ISBN10: 0125588402, pp: 340.

Qi, F. and B.-N. Guo, 2009. Two new proofs of the complete monotonicity of a function involving the psi function. Classical Analysis ODEs, 47: 103111. DOI: 10.4134/BKMS.2010.47.1.103

Sabanal, S. and M. Nakagawa, 1995. A study of timedependent fractal dimensions of vocal sounds, J. Phys. Soc. Jpn., 64: 3226. DOI: 10.1143/JPSJ.64.3226

Samko, S., A. Kilbas and O. Marichev, 1993. Fractional Integrals and Derivatives: Theory and Applications. 1st Edn., Gordon and Breach Science Publishers, New York, ISBN-10: 2881248640, pp: 976. 